\title{
Positronium in Phase IV and Rotator Phase of Long-Chain Alkanes
}

\author{
B. ZGARDZIŃSKA* AND T. GOWOREK \\ Lublin Positron Group (UMCS) \\ pl. Marii Curie-Skłodowskiej 1, 20-031 Lublin, Poland
}

\begin{abstract}
Ortho-positronium lifetime and intensity was measured as a function of temperature and pressure for some $n$-alkanes in which so-called "phase IV" appears. This phase is characterized by slightly increased ortho-positronium lifetime compared to low-temperature phases. It was found that the effect of quenching positronium by light does not appear in all phases from phase IV upwards. The lifetime in the rotator phases becomes equal to that in liquids, and only the change of positronium formation intensity allows to detect the solid-liquid transition in positron lifetime measurements.
\end{abstract}

PACS numbers: 36.10.Dr, 61.80.Fe, 71.60.+z, 78.70.Bj

\section{Introduction}

Sensitivity of $o$-Ps lifetime to the size of free volumes in solids allows to distinguish various structural phases. As an example one can give the phases in saturated hydrocarbons (alkanes, $\mathrm{C}_{n} \mathrm{H}_{2 n+2}$ ). The longer the chain is - the more solid phases appear. Beside ubiquitous rotator phase, for odd-numbered alkanes with $n \geq 25$ one can observe a phase abundant in end-gauche conformers (phase IV [1]). This paper is devoted to the study of decay of ortho-positronium (ortho-Ps, o-Ps) trapped in free volumes in the high-temperature solid phases of alkanes by positron annihilation lifetime spectroscopy (PALS). Among the longchain alkanes we describe below the results for two typical odd-numbered species with $n=25$ and $n=31$.

\section{Experimental}

Molten sample was placed in a chamber with positron source fixed in the center $\left(0.5 \mathrm{MBq}\right.$ of ${ }^{22} \mathrm{Na}$ in a kapton envelope). The sample was degassed by

*corresponding author; e-mail: bozena@hektor.umcs.lublin.pl 
freeze-thaw technique in order to avoid ortho-para conversion of positronium in dissolved molecular oxygen. In the measurements at high pressure the solid samples in the form of pellets (with $\mathrm{e}^{+}$source between them) filled a small tube closed by two pistons. Such a set was placed in the pressure chamber of Unipress U-11 gas compressor. The PALS spectra were measured using a standard fast-slow delayed coincidence setup. We assumed that the spectra consist of three exponential components convoluted with the instrumental resolution curve plus constant random coincidence background. Instrumental curve was approximated by a single Gaussian of 250 ps FWHM. The longest-lived of the three components was ascribed to the decay of ortho-Ps. The spectra were processed by the LT program [2]. To reduce the number of fitting parameters in LT analysis the intensity ratio paraPs to ortho-Ps was fixed, $I_{1}: I_{3}=1: 3$, according to the statistical weights of these states. A correction for positron absorption in kapton source envelope was also applied. Temperature was controlled with an accuracy $\pm 0.1 \mathrm{~K}$, pressure $\pm 0.5 \mathrm{MPa}$.

\section{Results and discussion}

Below we present the results of measurements for two alkanes: $n$-pentacosane $\mathrm{C}_{25} \mathrm{H}_{52}$ and $n$-hentriacontane $\mathrm{C}_{31} \mathrm{H}_{64}$. Figure 1 shows the temperature dependence of $o$-Ps lifetime $\tau_{3}$ and intensity $I_{3}$ in $n$-pentacosane. In the series of measurements with rising temperature the phase IV of $n$-pentacosane is difficult to observe due to very narrow range of its appearance, $0.7 \mathrm{~K}[3]$. However, in the cooling run the phase transitions show the hysteresis; "undercooling" of the transition rotator phase IV is much smaller than that of phase IV - low temperature phases; this way the range of existence of phase IV becomes extended to at least $3 \mathrm{~K}$. It is seen that in the region of phase IV the $o$-Ps lifetime is by about $200 \mathrm{ps}$ longer than in neighboring rigid phase. Such a lengthening of lifetime is expected when the molecular chain is shortened due to gauche location of end $\mathrm{C}-\mathrm{C}$ bond.

Since early '90s, it is known that the probability of positronium formation in polymers and some crystalline organic media rises in time due to accumulation of excess electrons in intermolecular traps [4]. This effect appears also in $n$-alkanes, and is particularly strong in the range of low temperatures; to some extent it manifests itself in rigid phase at the temperatures up to $328 \mathrm{~K}$ [5]. It seemed interesting to check if such an $I_{3}$ instability occurs in the phase IV. Thus, the measurements of type $\tau_{3}(T)$ and $I_{3}(T)$ were performed twice: with the samples in darkness and with illumination by LEDs (illumination throws out the electrons from the traps and restores the initial, stable, $I_{3}$ value). The maximum of trapped electron effect appears at about $200 \mathrm{~K}$ and diminishes with increasing the temperature. Figures 1 and 2 show that in phase IV there is no difference in o-Ps intensity with and without illumination, it means that excess electron trapping does not occur in that phase.

The phase transitions can be induced not only by temperature, but also by high pressure. In alkanes the rise of pressure is equivalent to decreasing the 


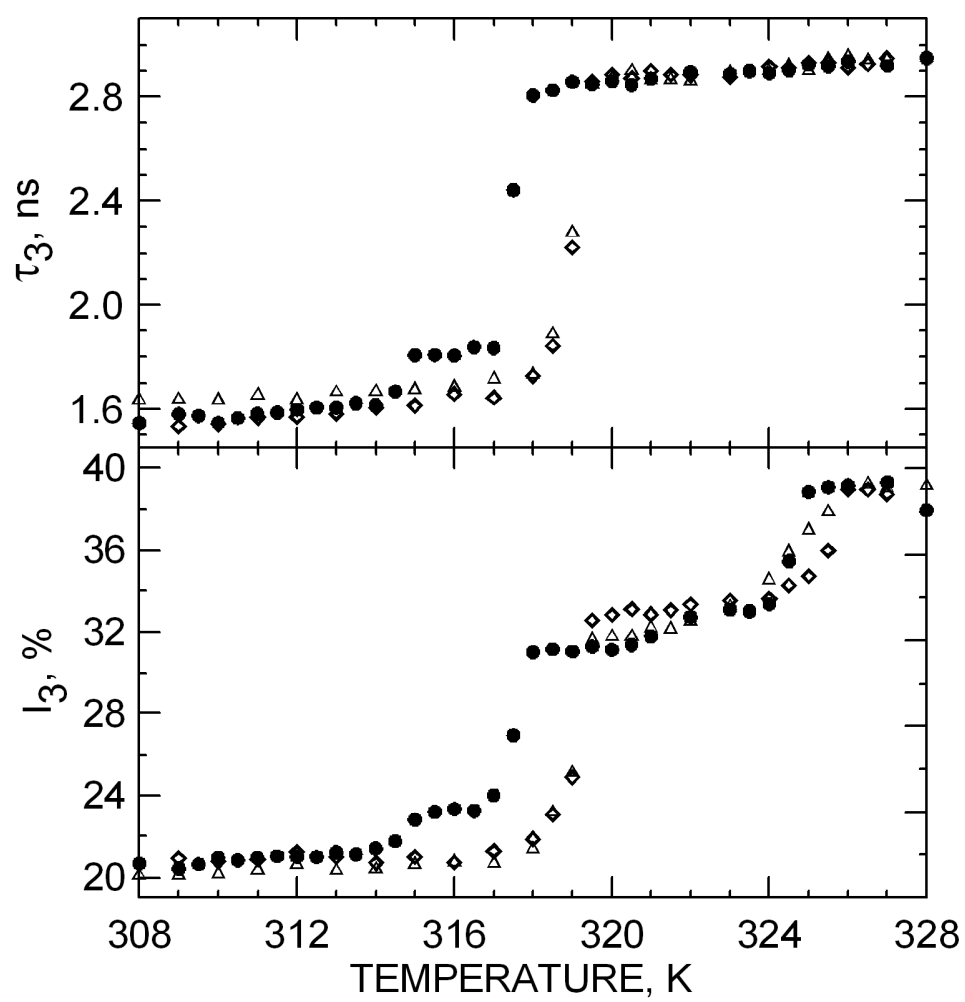

Fig. 1. Ortho-Ps lifetime and intensity in $n$-pentacosane. Triangles - temperature up, samples in darkness; diamonds - temperature up, samples illuminated by LED $0.95 \mu \mathrm{m}$; full circles — temperature down, illuminated.

temperature. We wanted to check if the hysteresis peculiarities seen in temperature dependences are reproduced in pressure dependences. The sample annealed in the rotator phase was cooled to $316 \mathrm{~K}$ and at that temperature the pressure dependence of $o$-Ps lifetime and intensity was measured. The result is shown in Fig. 3. Initially the sample was in phase IV and the transition to rigid phase can be seen at $10 \mathrm{MPa}$. After reduction of pressure to $0.1 \mathrm{MPa}$ the sample did not return to phase IV, like at increasing the temperature, i.e. the range of phase IV becomes again very narrow. The rotator phase is easily recognizable from rigid one as the $o-$ Ps lifetime in rotator exceeds $2 \mathrm{~ns}$, while in the rigid phase is rather close to $1.4 \mathrm{~ns}$. With increasing length of carbon chain, the $\tau_{3}$ lifetime in rotator rises and for the chains exceeding 23 carbon atoms becomes equal to the lifetime characteristic of liquids (about $3 \mathrm{~ns}$ ). It is possible to observe the transition rotator-liquid in the temperature dependence of $o$-Ps intensity only, as can be seen in Figs. 1 and 2.

With extending the carbon chain the range of phase IV increases and it can be observed in the heating runs, too. As an example of $n$-alkane with well pronounced phase IV can serve $n$-hentriacontane $\mathrm{C}_{31} \mathrm{H}_{64}$. The result of PALS mea- 


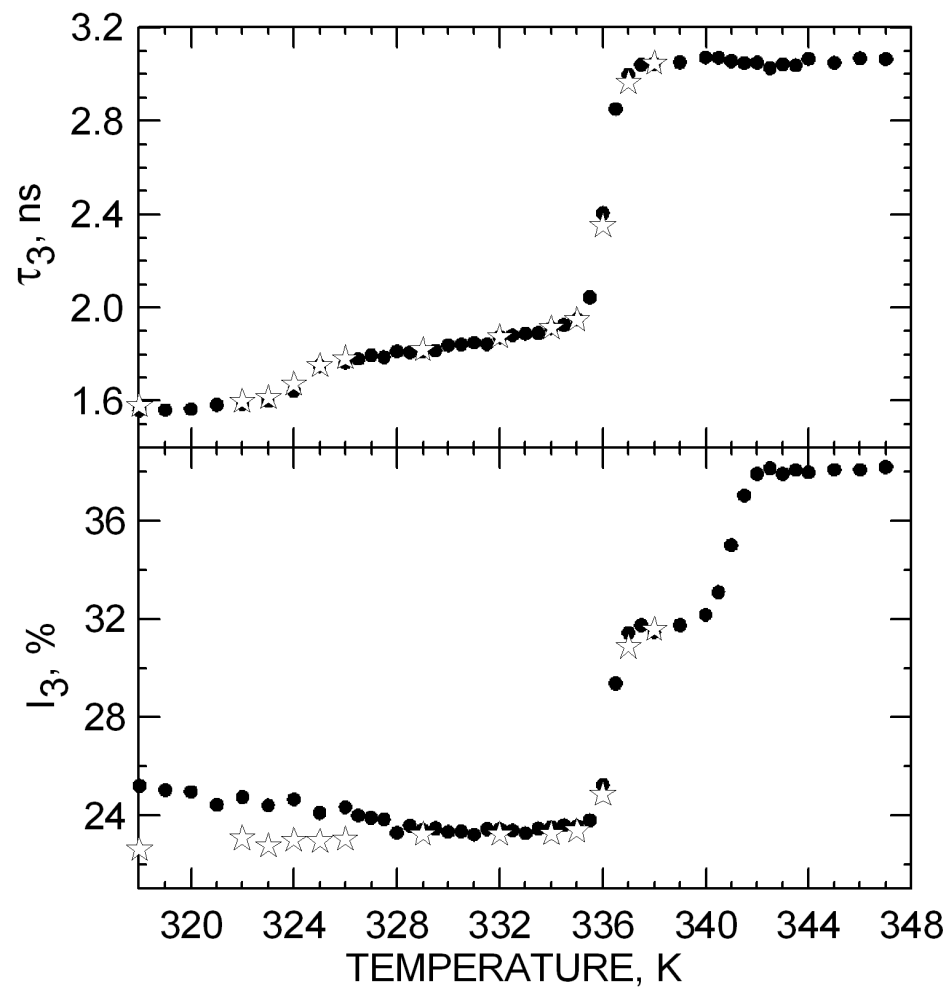

Fig. 2. Ortho-Ps lifetime and intensity in $n$-hentriacontane. Temperature up. Full symbols — sample in darkness, stars — sample illuminated by LED $0.95 \mu \mathrm{m}$.

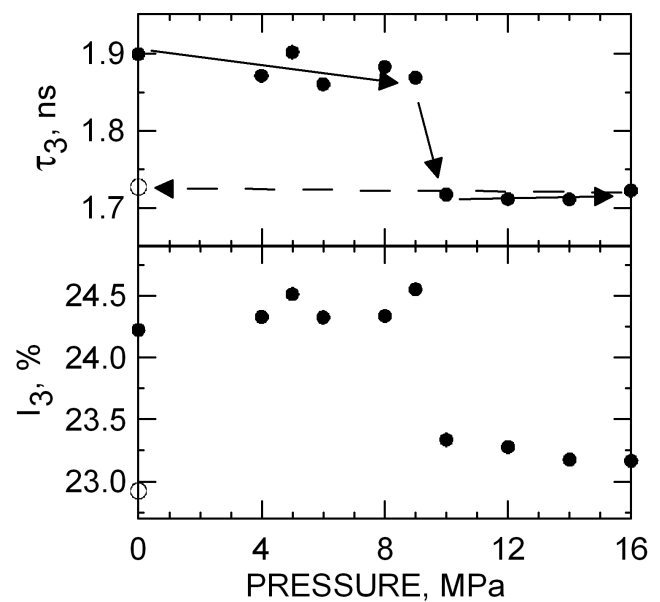

Fig. 3. Pressure dependence of $o$-Ps lifetime and intensity in $n$-pentacosane at the temperature $316 \mathrm{~K}$. 
surement for this alkane is shown in Fig. 2. Like in $n$-pentacosane, the phase IV is characterized by o-Ps lifetime lengthened by about $200 \mathrm{ps}$. The range of phase IV is now $11 \mathrm{~K}$; transition temperature is $324 \mathrm{~K}$, consistent with the data from Ref. [3]. Observation of such phase transitions for the chains longer than 31 carbons is difficult due to the tendency of forming a glassy structure after sample solidification.

As a rule, the phase transitions with very small transition enthalpy are accompanied by small changes of intermolecular free volumes and become difficult to observe in PALS measurements. For example, in $n$-pentacosane we have two rotator phases, but in both of them the lifetimes and intensities of $o$-Ps are, in the limits of error, identical. In $n$-hentriacontane there should be even three rotator phases (not observed experimentally yet; by extrapolation of data from Ref. [6] one can expect that two of them disappear at the next odd-numbered alkane). Also, the phase $\mathrm{V}$ is hard to notice in PALS measurements; its transition enthalpy is $0.3 \mathrm{~kJ} / \mathrm{mol}$, by order of magnitude smaller than that of transition to phase IV, and by two orders than for transition to rotator phase [3].

\section{Acknowledgments}

This work was supported by the Polish Ministry of Research and Academic Education grant N202 181 31/3928. Authors thank the Institute of Physics UMCS for making the equipment available for our measurements.

\section{References}

[1] T. Oyama, K. Takamizawa, Y. Ogawa, Kobunshi Ronbunshu 37, 711 (1980) (in Japanese).

[2] J. Kansy, Nucl. Instrum. Methods Phys. Res. A 374, 235 (1996).

[3] M. Maroncelli, Song Ping Qi, H.L. Strauss, R.G. Snyder, J. Am. Chem. Soc. 104, 6237 (1982).

[4] T. Hirade, F.H.J. Maurer, M. Eldrup, Radiat. Phys. Chem. 58, 465 (2000).

[5] T. Goworek, J. Wawryszczuk, R. Zaleski, B. Zgardzińska, Radiat. Phys. Chem. 76, 185 (2007).

[6] J. Doucet, I. Denicolò, A.F. Craievich, C. Germain, J. Chem. Phys. 80, 1647 (1980). 